\title{
The impact of integrating environmental health into medical school curricula: a survey-based study
}

\author{
Benjamin Kligler ${ }^{1}$, Genevieve Pinto Zipp ${ }^{2}$, Carmela Rocchetti ${ }^{3}$, Michelle Secic ${ }^{4}$ and Erin Speiser Ihde ${ }^{1 *}$ (D)
}

\begin{abstract}
Background: Inclusion of environmental health (EH) in medical education serves as a catalyst for preparing future physicians to address issues as complex as climate change and health, water pollution and lead contamination. However, previous research has found EH education to be largely lacking in U.S. medical education, putting future physicians at risk of not having the expertise to address patients' environmental illnesses, nor speak to prevention.

Methods: Environmental health (EH) knowledge and skills were incorporated into the first-year medical school curriculum at Hackensack Meridian School of Medicine (Nutley, New Jersey), via a two-hour interactive large group learning module with follow up activities. Students completed the Environmental Health in Med School (EHMS) survey before and after the year $1 \mathrm{EH}$ module. This survey evaluates medical students' attitudes, awareness and professionalism regarding environmental health. In year 2, students completed the Environmental Health Survey II, which measured students' perceptions of preparedness to discuss EH with future patients. The research team created both surveys based upon learning objectives that broadly aligned with the Institute of Medicine six competency-based environmental health learning objectives.
\end{abstract}

Results: 36 year 1 students completed both the pre and post EHMS surveys. McNemar's test was used for paired comparisons. Results identified no statistically significant changes from pre to post surveys, identifying a dramatic ceiling.

When comparing year 2, EHS II pre-survey $(n=84)$ and post-survey $(n=79)$ responses, a statistically significant positive change in students' self-reported sense of preparedness to discuss environmental health with their patients following the curriculum intervention was noted.

Conclusions: Our conclusion for the EHMS in Year 1 was that the current generation of medical students at this school is already extremely aware of and concerned about the impact of environmental issues on health. Through the EHS II in Year 2, we found that the six-week environmental health module combining didactic and experiential elements significantly increased medical students' self-reported sense of preparedness to discuss environmental health issues, including climate change, with their patients.

Keywords: Environmental health, Medical education, Prevention, Environmental justice, Health disparities, Climate change

\footnotetext{
* Correspondence: erin.ihde@hmhn.org

${ }^{1}$ The Deirdre Imus Environmental Health Center ${ }^{\oplus}$, Hackensack University Medical Center, Hackensack, NJ, USA

Full list of author information is available at the end of the article
}

(c) The Author(s). 2021 Open Access This article is licensed under a Creative Commons Attribution 4.0 International License, which permits use, sharing, adaptation, distribution and reproduction in any medium or format, as long as you give appropriate credit to the original author(s) and the source, provide a link to the Creative Commons licence, and indicate if changes were made. The images or other third party material in this article are included in the article's Creative Commons licence, unless indicated otherwise in a credit line to the material. If material is not included in the article's Creative Commons licence and your intended use is not permitted by statutory regulation or exceeds the permitted use, you will need to obtain permission directly from the copyright holder. To view a copy of this licence, visit http://creativecommons.org/licenses/by/4.0/ The Creative Commons Public Domain Dedication waiver (http://creativecommons.org/publicdomain/zero/1.0/) applies to the data made available in this article, unless otherwise stated in a credit line to the data. 


\section{Background}

The World Health Organization estimates 23\% of global disease is attributable to environmental factors, which rises to $25 \%$ for children under age five [1]. Inclusion of environmental health (EH) in medical education serves as a catalyst for preparing future physicians to address issues as complex as climate change and health, water pollution and lead contamination in our communities [2-4]. However, previous research has found EH education to be largely lacking in U.S. medical education, putting future physicians at risk of not having the expertise necessary to address patients' environmental illnesses, nor speak to prevention [4-6]. This is of particular concern for future pediatricians, as children are especially vulnerable given their immature detoxification pathways and tendencies to play close to the ground where contaminants settle $[7,8]$. Other vulnerable populations include the elderly, those with pre-existing health conditions and marginalized populations. Environmental health disparities, therefore, fit well under the larger umbrella of social determinants of health, which are garnering increasing attention yet still remain largely omitted from medical school curricula [9-12].

As part of its commitment to addressing social determinants of health in the medical school curriculum, Hackensack Meridian School of Medicine (HMSOM) in Nutley, New Jersey developed, launched and tested a new environmental health module in the required first year curriculum. The goal was to produce an effective and reproducible curriculum which could be utilized by other medical schools to address the gap in environmental health knowledge among physicians.

\section{Methods}

The new curriculum module was incorporated into the Human Dimension (HD), a three-year longitudinal course at the heart of the HMSOM curriculum. Through service-learning experiences and an integrated curriculum, students understand the many Determinants of Health, including the social determinants of health as well as the personal, economic, and environmental determinants. Students are matched to individuals and families from underserved areas, and through longitudinal interactions over the entire core curriculum, they become involved in all aspects of the family's life to understand drivers of health outcomes, provide education, and navigate community resources. Activities include meetings and calls with individuals and families in their homes and communities as well as participation in multidisciplinary teams in health care, legal, and social services settings.

As part of this effort, the research team created an Environmental Health module delivered via a two-hour interactive large group learning module (lecture) with follow-up activities (a six-week-long student activity highlighting the ways in which the environment and medicine intersect and then a small group discussion session to process that experience). The lecture was titled, "Environmental Health: What Do Physicians Need to Know?" and given by BK. Lecture topics included the health effects of exposures to common toxins, avoiding carcinogens and endocrine disruptors, choosing healthier food and personal care products, the health impacts of climate change, and identifying populations at most risk for environmental health issues. The lecture included two small group discussion periods on questions of how to communicate effectively with patients regarding environmental health and how to begin to make changes in one's own behaviors. The activity had two components; first, students were asked to download the "Healthy Living" app produced by Environmental Working Group, which provides details on the environmental safety of a wide range of foods and personal care products. Students were asked to explore their diet and personal care choices by identifying 5-10 items and using the app to assess the relative safety of those products from an environmental health perspective. In addition, students were provided with a set of assessment tools to use to understand the potential environmental exposure risk of their HD family. The $\mathrm{EH}$ module is delivered midway through the first year of the HD curriculum, and both small group discussions and the communitybased work with the HD family is overseen by the HD course faculty who have received faculty development sessions on this topic.

The EH module was given during two subsequent years at the medical school. In year 1, the 15-question Environmental Health in Med School (EHMS) survey was given in January 2019 and the post was given in March 2019. This tool was developed by ESI, GPZ and BK. While there were approximately 60 first-year students invited to take the survey, there were 36 subjects who had data on both the pre and post surveys and were included in the statistical analysis.

For statistical analysis of the EHMS, data were summarized for each question both pre and post with counts and percentages. Responses on the pre survey were compared to those on the post survey using McNemar's test of agreement. For statistical purposes, results were collapsed as follows, excluding neutrals: True $=$ Definitely true/Somewhat true; False = Definitely false $/$ Somewhat false; Agree $=$ Strongly agree $/$ Agree; Disagree $=$ Strongly disagree/Disagree.

In year 2, students took a newly-created 4-question Environmental Health Survey II (EHS II) before their EH module in November 2019 and again as a post survey in March 2020. This tool was created by BK, who gave the $\mathrm{EH}$ lecture in the large group learning module. 
A total of 84 students took the pre survey, and 79 took the post.

The two surveys were designed to help inform subsequent revisions to the module content to ensure learning objectives were met. These objectives broadly align with the six competency-based environmental health learning objectives from the Institute of Medicine [13].

The Year 1 EHMS survey was designed to evaluate medical students' attitudes, awareness and professionalism regarding environmental health. Each of the three constructs that were measured had five corresponding questions in the survey, for a total of 15 questions. A Delphi panel of five experts with backgrounds in curriculum design were asked to rate each question based on three criteria: Is the question appropriate for this survey, is the question clearly written, and is the question in the correct sequence? Each of these had yes/no response options followed by "If no, what are your suggestions for improvement?"

A total of two rounds of the Delphi process were conducted until at least $80 \%$ consensus was reached on each question. To control the validity issues associated with the Delphi technique, the survey questions were revised to provide clarity where needed. Based on this first survey, a new survey (EHS II) was created for Year 2 of new, incoming medical students. This brief survey was designed to measure students' perceptions of preparedness to discuss $\mathrm{EH}$ with future patients as opposed to their attitudes, awareness, and professionalism. There were no tests of reliability or validity for this EHSII. The questions were: 1) I feel prepared to advise my patients on strategies for minimizing exposure to pesticides and other environmental toxins in their diet. 2) I feel prepared to advise my patients on strategies for minimizing exposure to environmental toxins in their household and personal care products. 3) I know enough to advocate in my community to try to reduce the healthrelated impact of environmental toxins on air and water quality. 4) I feel prepared to discuss the specific impacts of climate change on human health. Response options were organized into a 5-point Likert scale ranging from "totally disagree" to "totally agree." For the EHSII, the Wilcoxon signed-rank test was used to ascertain whether the EH module was effective in promoting medical students' preparedness to discuss environmental health with their patients.

Data from both surveys was collected nonanonymously using a secure system. To protect participants' confidentiality, only the HMSOM Institutional Effectiveness and Assessment team had access to identifying information. They provided de-identified data to the research team, including unique identifiers created solely for this project to link the pre and post survey responses. The pre and post surveys fell under the written consent already obtained from the students for LongMED, a database that supports an epidemiologic, longitudinal, outcomes-focused approach to the study of medical education. LongMED contains protected, linked data tied to medical students at the HMSOM.

All students completed the pre and post surveys as part of the curriculum, but the HMSOM sent the research team only the responses for the students who consented to participate in LongMED. Students' interaction with the instructor and their grade in the HD course were not affected in any way by taking or declining to take the pre and post survey. All first-year medical students (approximately 60 in Year 1 and 85 in Year 2) at the HMSOM, who consented to use LongMED and attended the HD Module, were invited to participate. The final study protocol, survey and data collection tool were approved by the Institutional Review Board (IRB) at Hackensack University Medical Center, Hackensack, New Jersey, USA.

The specific content of the lecture including PowerPoint slides as well as detailed descriptions of the other elements in the EH module are available as Additional files 1 and 2 to this paper.

\section{Results}

For the EHMS in Year 1, 36 subjects had data on both the Pre and Post surveys and thus were eligible for the paired comparisons. In the $2 \times 2$ cross-tabulations, most subjects did not change their answers at all or significantly from pre to post. For example, for Q1, 4 subjects answered true on the Pre survey and all 4 of those answered false on the Post survey; 25 subjects answered false on the Pre survey and 23 of those answered false on the Post survey, while 2 of them answered true on the Post survey. Although 4 switched from true to false and 2 switched from false to true, the differences in these changes were not statistically significant, $p=0.41$.

Therefore, no statistically significant changes from Pre to Post were found, using the standard two-tailed alphalevel of 0.05 . Results of the pre and post for the first survey suggested a dramatic ceiling effect on students' responses to the EHMS survey, making it difficult to detect any change from before to after the intervention.

For the Year 2 survey (EHS II), Fig. 1 demonstrates the percentage of respondents across the 5-point Likert scale ranging from "totally disagree" to "totally agree" for the four questions on the pre-survey (84 respondents) and on the post-survey (79 respondents). As reported in Table 1, the Wilcoxon test showed a statistically significant positive change for each question posed in students' self-reported sense of preparedness to discuss environmental health with their patients following the curriculum intervention. 
I feel prepared to advise my patients on strategies for minimizing exposure to pesticides and other environmental toxins in their diet.

I feel prepared to advise my patients on strategies for minimizing exposure to environmental toxins in their household and personal care products.

I know enough to advocate in my community to try to reduce the health-related impact of environmental toxins on air and water quality.

I feel prepared to discuss the specific impacts of climate change on human health.
$21 \%$

\section{$59 \%$}

$24 \%$

$58 \%$

$14 \%$

$56 \%$

$33 \%$ $59 \%$

$25 \%$

$50 \%$

$75 \%$

$100 \%$

Pre-Survey Post-Survey

Fig. 1 Percentage of respondents in each administration that responded 'Totally Agree' or 'Somewhat Agree' to the four EHS II questions, $n=84$ on pre-survey (dark blue) and $n=79$ on post-survey (light blue)

\section{Discussion}

As early as 1995, the Institute of Medicine (IOM) recommended six competency-based $\mathrm{EH}$ learning objectives for medical students, as shown in Table 2:

Despite these recommendations, though, and the growing awareness of the health impacts of climate change and environmental degradation, there is no standard requirement to include EH coursework in medical schools. To our knowledge this is the first evaluation and description of an environmental health curriculum intervention which demonstrates significant change in students' sense of preparedness to discuss EH with their patients.

Although several medical schools do offer some exposure to $\mathrm{EH}$, including Harvard and Mount Sinai, there is a general sense that finding time in the curriculum for this subject matter is still difficult [14]. Climate change in particular is apparently being overlooked: a survey of the Curriculum Inventory of the American Association of Medical Colleges in 2017 revealed no explicit mention of climate change in any medical school curriculum [15] though efforts to increase climate change in medical curricula now exist [14]. The lack of such curriculum continues to lead to a sense of unpreparedness in providers to discuss this area with patients: a 2010 survey of pediatric providers reported a lack of training in environmental history taking and low self-efficacy in how to manage patients' exposures [7]. A similar survey of pediatric oncologists found that over $90 \%$ of respondents felt that "more knowledge of the associations between environmental exposures and childhood cancer would be helpful in addressing these issues with patients" [16].

Integrating $\mathrm{EH}$ material into existing courses - as we have done at the HMSOM - may be more feasible than restructuring an already dense curriculum to include dedicated EH courses [5, 17]. Distance-based education

Table 1 EHS II Means (SD) Analysis, Pre-Survey $(n=84)$ v. Post-Survey $(n=79)$

\begin{tabular}{|c|c|c|c|}
\hline Responses & Pre-Survey & Post-Survey & Wilcoxon Signed-Rank Test $P$-Value \\
\hline $\begin{array}{l}\text { I feel prepared to advise my patients on strategies for minimizing exposure } \\
\text { to pesticides and other environmental toxins in their diet. }\end{array}$ & $2.31(1.086)$ & $3.46(0.958)$ & $<0.001$ \\
\hline $\begin{array}{l}\text { I feel prepared to advise my patients on strategies for minimizing exposure } \\
\text { to environmental toxins in their household and personal care products. }\end{array}$ & $2.48(1.081)$ & $3.47(0.918)$ & $<0.001$ \\
\hline $\begin{array}{l}\text { I know enough to advocate in my community to try to reduce the } \\
\text { health-related impact of environmental toxins on air and water quality. }\end{array}$ & $2.27(0.986)$ & $3.39(1.005)$ & $<0.001$ \\
\hline I feel prepared to discuss the specific impacts of climate change on & $2.80(1.210)$ & $3.46(1.095)$ & $<0.001$ \\
\hline
\end{tabular}


Table 2 Institute of Medicine Competency Based Environmental Health Learning Objectives for Medical Students

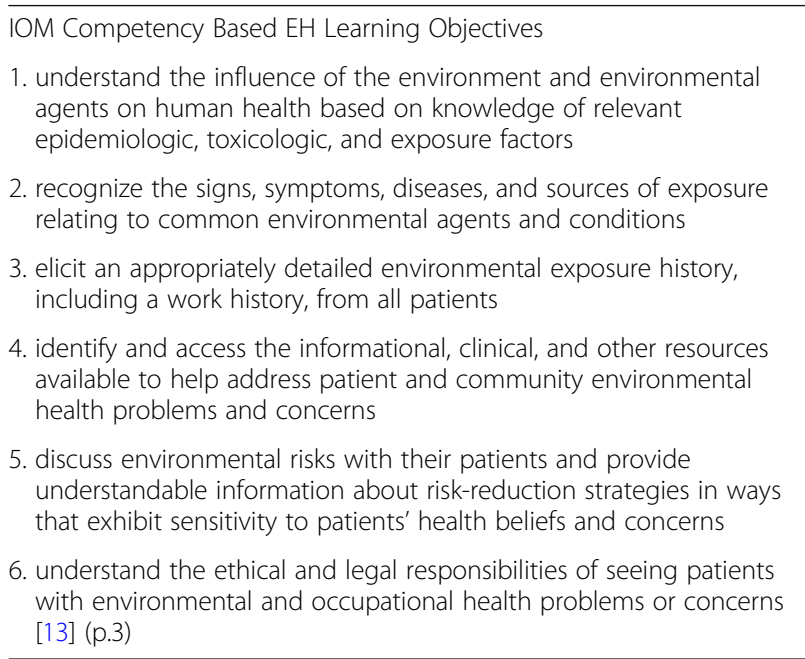

and engagement of basic science faculty to teach some competencies may help alleviate the shortage of faculty trained in environmental medicine [5]. A new multimedia e-book developed by Miller et al. and endorsed by the Centers for Disease Control and Prevention (CDC) "A Story of Health" - is an excellent resource as well, especially where local faculty expertise in EH may be lacking [18]. Finally, it is also important to combine didactic with experiential and small-group learning as we have done, particularly when the goal is to impact students' sense of preparedness and self-efficacy around discussing $\mathrm{EH}$ with patients. Experience discussing this issue with colleagues and experimenting with actually making behavioral changes in their own lives are also critical.

\section{Limitations}

A limitation in this study is the use of self-assessed outcomes rather than objective observation. This could be addressed by incorporating $\mathrm{EH}$ concepts into a standardized patient assessment experience where those resources are available. A second limitation of this study is that we present results of the preparedness survey from only one cadre of medical students. Further research on the impact of incorporating environmental health into required curriculum is needed, especially on the reproducibility of this particular module in increasing competency for medical student learners in this important area both at HMSOM and at other medical schools that may utilize this curriculum.

\section{Conclusions}

The EHMS in Year 1 suggested the current generation of medical students at this school is already extremely aware of and concerned about the impact of environmental issues on health. Analysis of the EHS II in Year 2 showed that a relatively brief six-week environmental health module combining didactic and experiential elements can significantly increase medical students' selfreported sense of preparedness to discuss environmental health issues, including climate change, with their patients.

\section{Supplementary Information}

The online version contains supplementary material available at https://doi. org/10.1186/s12909-020-02458-x.

\section{Additional file 1.}

Additional file 2.

\section{Abbreviations}

CDC: Centers for Disease Control and Prevention; EH: Environmental health; EHMS: Environmental Health in Medical School; EHS II: Environmental Health Survey II; HMSOM: Hackensack Meridian School of Medicine; HD: Human Dimension

\section{Acknowledgements}

The authors wish to thank Christopher P. Bujara and Jesse M. Jacondin of the Hackensack Meridian School of Medicine (HMSOM), for assisting with the survey and preparing the data for this study. The authors also thank LaTanya M. Brown and Michel'le J. Bryant at the HMSOM for assisting with coordinating the HD module.

\section{Authors' contributions}

BK participated in the study design, and contributed medical curriculum expertise and detailed manuscript review. CR participated in the curriculum design and manuscript review. GZ participated in the study design and oversight and detailed manuscript review. MS conducted the statistical analysis of the EHMS survey and reviewed the manuscript. ESI participated in the study design, coordinated study activities and drafted the manuscript. The author(s) read and approved the final manuscript.

\section{Funding}

There are no funding sources to declare.

\section{Availability of data and materials}

The de-identified data sets generated and analyzed during the current study will be made available from the corresponding author, on reasonable request, to any scientist wishing to use them for non-commercial purposes. The authors are also happy to share the detailed content of the presentations and small group activities included in this module.

\section{Ethics approval and consent to participate}

This research study was conducted in accordance with the Declaration of Helsinki. Written consent was obtained from study participants. The final study protocol, survey and data collection tools were approved prior to the commencement by the Institutional Review Board (IRB) at Hackensack University Medical Center, Hackensack, New Jersey, USA via Pro\# 2018-0974.

\section{Consent for publication}

All authors read and approved the final manuscript.

\section{Competing interests}

The authors declare that they have no competing interests.

\section{Author details}

${ }^{1}$ The Deirdre Imus Environmental Health Center ${ }^{\circledast}$, Hackensack University Medical Center, Hackensack, NJ, USA. ²Department of Interprofessional Health Sciences \& Health Administration, School of Health and Medical Sciences, Seton Hall University, Nutley, NJ, USA. ${ }^{3}$ Hackensack Meridian School of Medicine, Nutley, NJ, USA. ${ }^{4}$ Secic Statistical Consulting, Inc., Chardon, $\mathrm{OH}$, USA. 
Received: 13 July 2020 Accepted: 16 December 2020

Published online: 08 January 2021

\section{References}

1. Folkl A, Malgeri M. Environmental health: how much do medical students know? EducPrim Care. 2010;21(3):194-5. https://doi.org/10.1080/14739879. 2010.11493905.

2. Garg A, Mulloy KB. Developing a problem-based learning approach to the integration of environmental and occupational health topics into medical school curriculum. J Occup Environ Med. 2018;60(8):754-9. https://doi.org/ 10.1097/JOM.0000000000001325.

3. Prüss-Üstün A, Wolf J, Corvalán C, Bos R, Neira M. Preventing disease through healthy environments: a global assessment of the burden of disease from environmental risks: World Health Organization; 2016. Available from: https://apps.who.int/iris/handle/10665/204585 / ISBN: 9789241565196.

4. Roberts JR, Reigart JR. Environmental health education in the medical school curriculum. Ambul Pediatr. 2001;1(2):108-11. https://doi.org/10.1367/ 1539-4409(2001)001<0108:eheitm>2.0.co;2.

5. Gehle KS, Crawford JL, Hatcher MT. Integrating environmental health into medical education. Am J PrevMed. 2011;41(4 Suppl 3):S296-301. https://doi. org/10.1016/j.amepre.2011.06.007

6. Tinney VA, Paulson JA, Bathgate SL, Larsen JW. Medical education for obstetricians and gynecologists should incorporate environmental health. Am J Obstet Gynecol. 2015;212(2):163-6.e1. https://doi.org/10.1016/j.ajog. 2014.07.038

7. Trasande L, Newman N, Long L, Howe G, Kerwin BJ, Martin RJ, Gahagan SA, Weil WB. Translating knowledge about environmental health to practitioners: are we doing enough? Mt Sinai J Med. 2010;77(1):114-23. https://doi.org/10.1002/msj.20158.

8. McCurdy LE, Roberts J, Rogers B, Love R, Etzel R, Paulson J, Witherspoon NO, Dearry A. Incorporating environmental health into pediatric medical and nursing education. Environ Health Perspect. 2004;112(17):1755-60. https:// doi.org/10.1289/ehp.7166.

9. Basu G, Pels RJ, Stark RL, Jain P, Bor DH, McCormick D. Training internal medicine residents in social medicine and research-based health advocacy: a novel, in-depth curriculum. Acad Med. 2017;92(4):515-20. https://doi.org/ 10.1097/ACM.0000000000001580

10. Campbell-Yesufu M. Developing a community-centered curriculum on social determinants of health. Acad Med. 2018;93(6):818-9. https://doi.org/ 10.1097/ACM.0000000000002199.

11. Kasper J, Greene JA, Farmer PE, Jones DS. All health is global health, all medicine is social medicine: integrating the social sciences into the preclinical curriculum. Acad Med. 2016;91(5):628-32. https://doi.org/10.1097/ ACM.0000000000001054

12. Peabody JW. Measuring the social responsiveness of medical schools: setting the standards. Acad Med. 1999;74(8 Suppl):S59-68. https://doi.org/ 10.1097/00001888-199908000-00031.

13. Institute of Medicine (US) Committee on Curriculum Development in Environmental Medicine. In: Pope AM, Rall DP, editors. Environmental Medicine: Integrating a Missing Element into Medical Education. Washington (DC): National Academies Press (US); 1995.

14. Friedrich MJ. Medical community gathers steam to tackle climate's health effects. JAMA. 2017;317(15):1511-3. https://doi.org/10.1001/jama.2017.0969.

15. Wellbery C, Sheffield P, Timmireddy K, Sarfaty M, Teherani A, Fallar R. It's time for medical schools to introduce climate change into their curricula. Acad Med. 2018;93(12):1774-7. https://doi.org/10.1097/ACM. 0000000000002368.

16. Zachek CM, Miller MD, Hsu C, Schiffman JD, Sallan S, Metayer C, Dahl GV. Children's cancer and environmental exposures: professional attitudes and practices. J Pediatr Hematol Oncol. 2015;37(7):491-7. https://doi.org/10. 1097/MPH.0000000000000416.
17. Walpole SC, Vyas A, Maxwell J, Canny BJ, Woollard R, Wellbery C, LeedhamGreen KE, Musaeus P, Tufail-Hanif U, Pavão Patrício K, Rother HA. Building an environmentally accountable medical curriculum through international collaboration. Med Teach. 2017;39(10):1040-50. https://doi.org/10.1080/ 0142159X.2017.1342031.

18. Miller MD, Valenti M, Schettler T, Tencza B. A multimedia e-book-a story of health: filling a gap in environmental health literacy for health professionals. Environ Health Perspect. 2016;124(8):A133-6. https://doi.org/10.1289/ EHP222.

\section{Publisher's Note}

Springer Nature remains neutral with regard to jurisdictional claims in published maps and institutional affiliations.
Ready to submit your research? Choose BMC and benefit from:

- fast, convenient online submission

- thorough peer review by experienced researchers in your field

- rapid publication on acceptance

- support for research data, including large and complex data types

- gold Open Access which fosters wider collaboration and increased citations

- maximum visibility for your research: over $100 \mathrm{M}$ website views per year

At $\mathrm{BMC}$, research is always in progress.

Learn more biomedcentral.com/submissions 